\title{
Artificial Induction of Meiotic Chromosome Pairing in the Somatic Cell of Drosophila virilis ${ }^{1}$ (Cytological studies on D. virilis, Pt. III) ${ }^{2}$

\author{
By
} \\ Sajiro Makino \\ Zoological Institute, Faculty of Seience, Hokkaido Imperial University, Sapporo
}

(With 9 Figures)

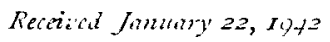

The question of how the pairing of the homologous chromosomes is brought about in meiosis has been a matter of repeated discussions in recent cytology and various theories have been advanced to explain the chromosome behaviour observed in meiosis, particularly as to the cause and mechanism of chromosome pairing. Of special importance are the precocity theory put forward by Darlington ('32, '37), the unified hypothesis emphasized by Huskins ('33) and the retardation theory proposed by Sax \& Sax ('35). The former two hold the principle that chromosome pairing and synapsis at meiosis is dependent upon the univalency of the synapting chromosomes. The retardation theory points out that meiosis results from a retardation of cellular activity, being based on the evidences indicating that a much longer time is required for the process of meiosis than for mitosis. Recently an important experimental demonstration has been made by Matsuura \& Haga ('40) in the work with Trillium, showing that by artificially speeding up the division process, meiosis is convertible into mitosis, an evidence which favors in its interpretation the retardation theory. There is, however, no reference in the literature to the artificial induction of meiosis in the somatic cell. The present investigation succeeded in the demonstration of meiotic pairing of chromosomes in somatic cells of Drosophila virilis, by means of suppression of the cell division process leading to the prolongation of the resting stage in those cells, which show a pronounced similarity in the structural condition of the resting nucleus to the meiotic prophase nucleus by the evidence of the side-by-side association of the homologous chromosome threads ( $c f$. Makino' 40 ). Though the investigation may not be so fruitfully worked out, publishing this piece of work might contribute something to the analysis of the complex mechanism of meiosis.

1 Contribution No. 161 from the Zoological Institute, Faculty of Science, Hokkaido Imperial University, Sapporo, Japan.

2 Part I was published in Cytologia 9 (1938) and Part II in Cytologia 10 (1940). 
The author gratefully acknowledges the valuable suggestion offered by Prof. K. Oguma during the progress of this work. Thanks are also due to Prof. H. Matsuura for his helpful advice. A part of the expense of this work was defrayed out of a grant from The Japan Society for the Advancement of Cytology, through the courtesy of Prof. K. Fujii, the president of the society, to whom thanks are due.

\section{Method}

Normal wild-type flies of Drosophila virilis furnished from the stock of the Zool. Institute of Kyoto Imperial University through the courtesy of Dr. M. Chino, to whom the author's thanks are due, were employed in this investigation as in the previous studies. It has already been known that the oesophageal ganglia of larvae of the last instar just prior to pupate, reared under favourable condition (at $24-25^{\circ} \mathrm{C}$ ), contain in general many cells in the process of mitotic division. It is these ganglion cells, the neurocytes, of old larvae on which the present observations and experiments were carried out. With the hope to make persist the cells for a long time in the resting condition by the retardation of the division process, the vials containing old larvae are placed in a refrigerator, kept at a temperature of about $5^{\circ} \mathrm{C}$ for 2,3 and 5 days in order to prolong the larval period. Then the vials are left at room temperature for several hours prior to study. In another experiment, old larvae were reared with waste culture media at unfavourable low temperature.

For study of the cells temporary aceto-carmine smear preparations were employed. The ganglia were removed from the larvae and placed in a watch crystal containing aceto-carmine, for 1 hour or more prior to transferring to a slide. Then they were taken on a slide with a drop of aceto-carmine and covered with a cover glass. The cells were flattened by rolling a glass vial heavily weighted across the cover.

\section{Observations}

\section{Normal chromosome behavior in ganglion cells}

The chromosome group in the ganglion cell (neurocyte), as given in the previous paper (Makino '40), consists of five rod-like pairs and one small dot-like pair (Figs. 1-2). One of the rod-like pairs should be the sex-chromosome pair: the latter is made up of the $X$ and $Y$ elements in the male, and 2X's in the female. Since the $\mathrm{X}$ and $\mathrm{Y}$ elements exhibit no distinct visible difference in the morphological feature at metaphase between them, there is present no distinction in the chromosome complement between the male and female. The metaphase pattern of the chromosomes of both the sexes is thus quite similar in appearance, twelve elements being observable in either of them. Though Metz ('27) mentioned that the $\mathrm{X}$ and $\mathrm{Y}$ members slightly differ in size, the recent 
investigations show that his study is observationally invalid in this respect. The homologous chromosomes lie in close proximity to each other, every two arranging side by side.

At prophase there appear in the nucleus six groups of chromosome threads, five extremely long and one very short, radiating from a deeply stained, central chromatic concentration (Fig. 3). It was established in the author's previous study (Makino '40) that each group of chromosome threads consists of two distinct elements lying in side-by-side association, and that the elements constituting each group represent homologous members. The distal ends of all the threads are longitudinally split, their extremities being almost indefinable. The central chromatic concentration to which the threads converge is the chromocentre, an aggregate of the hetero-chromatic regions forming the proximal portions of each chromosomal element (Makino "40). It is specially noticeable that in the prophase nucleus of the somatic cell every two homologous mates of the chromosome elements associate side by side in close proximity, assuming a feature just comparable to that usual in the early prophase of meiosis. Therefore the number of these groups of the chromosome threads just corresponds to the haploid number of this species. For more detailed accounts on the behaviour of chromosomes during the mitotic cycle of the neurocyte reference should be made to the author's previous study (Makino' 40 ).

\section{Induced bivalent chromosomes in ganglion cells}

In the ganglia of old larrae reared under unfavourable conditions with the treatments as already mentioned, there are found, among many resting cells, unusual metaphase plates which contain six distinct chromosomes, five large with nearly equa! size and one extremely small, representing the haploid number of chromosomes. Fxamples of them are presented in Figs. 4, 5 and 6. The chromosomes as seen in these metaphase plates are quite remarkable in that they are provided with the shape of a ring lying horizontally on the equatorial plate, except one small element whose shape is difficult to define on account of its minute size. The lateral configuration of these chromosomes is illustrated in Fig. 8. The five large elements occupy the peripheral circle of the spindle with the small element in the central space. By the precise examination of these chromosomes either in the polar view or from the lateral side, it becomes evident that the structural feature of these ring-shaped chromosomes are fairly identical to the horizontal ring-shaped tetrad (bivalents) derived from telomitic homologous chromosomes. As a matter of fact, these ring-shaped elements show nothing different in appearance and structure from the ring tetrad generally observable in the first meiotic division of many species of acridian Orthoptera and small 
rodents such as mice and rats, all of which are characterized in having telomitic chromosomes. Viewed from such a structural configuration and also from the fact that the number of elements included in these equatorial plates fairly corresponds to the haploid number of this species, there is no doubt to conclude that they are bivalent chromosomes resulting
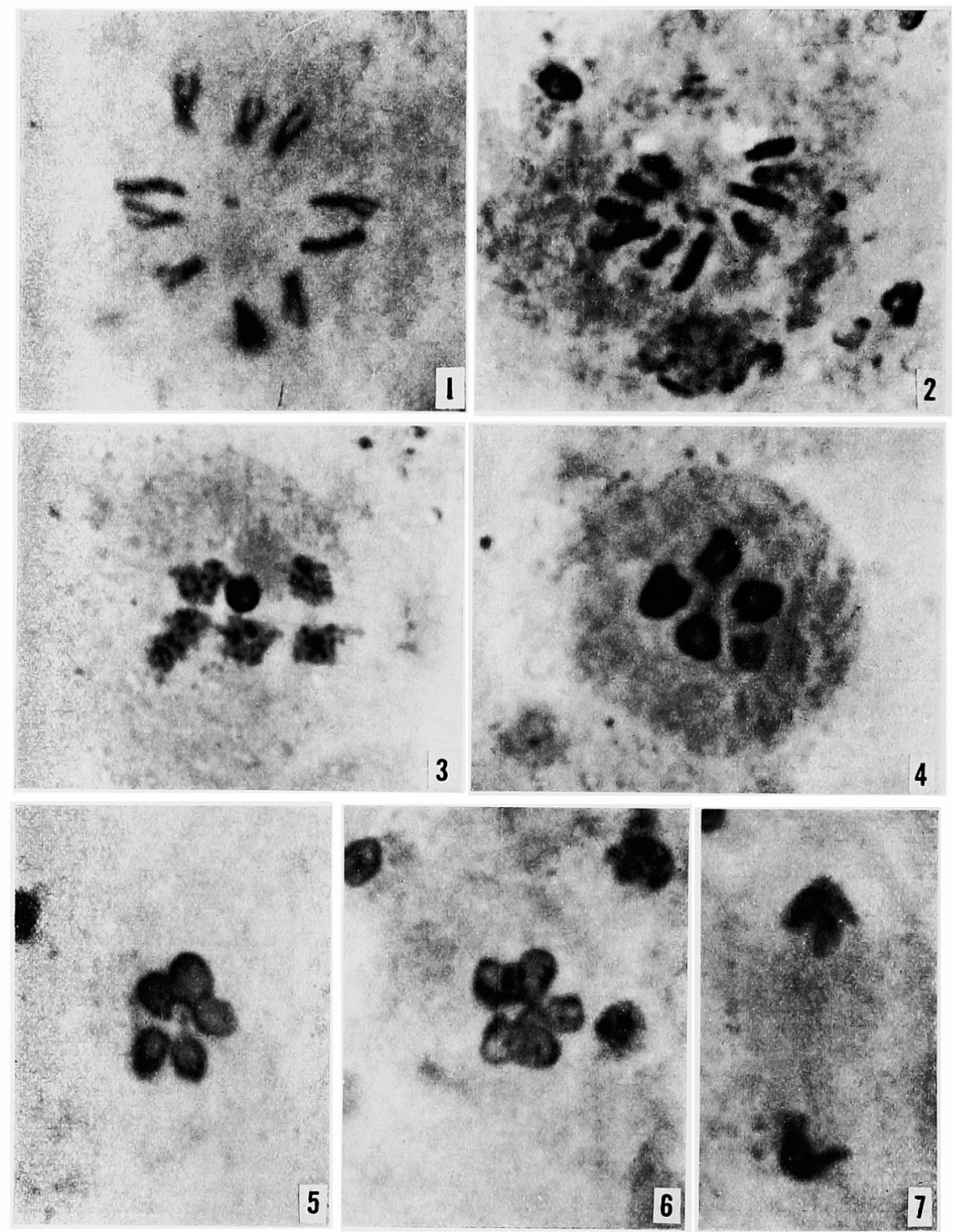

Figs. 1-2. Normal metaphase figures in neurocytes of larvae of $D$. virilis. 12 chromosomes in each. Fig. 3. Prophase nucleus of the same. Six groups of chromosome threads are seen converging to a central body, the chromocentre. Figs. 4-6. Metaphase figures showing bivalent chromosomes induced in neurocytes. Six bivalents, five large and one minute, are observable in each. Fig. 7. Telophase of the same. All are photomicrographs taken from aceto-carmine preparations. $\times 2800$. 
from the pairing of every two homologous mates.

Since the induced bivalent chromosomes are entirely regular in arrangement in the metaphase plate and the normal spindle is formed at metaphase, there is a strong possibility that the regular division may occur in these chromosomes at anaphase. Though it cannot be said with certainty, ocrasional occurrence of the haploid plate containing six univalent elements as shown in Fig. 9 in the ganglia of the treated larvae, may find its most farour-
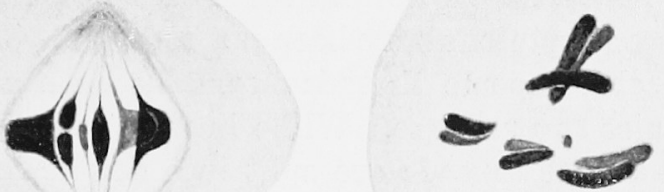

8

\section{9}

Fig. 8. Side view of induced bivalent chromosomes in a neurocy te of D.virilis. Fig. 9. Metaphase plate showing six univalent chromosomes in a neurocyte. Camera-lucida drawings. $\times 2400$. able explanation in

assuming that such haploid univalent chromosomes are produced as a result of the separation of bivalent chromosomes and that they correspond in nature, therefore, to the second metaphase chromosomes. As regards the destiny of these cells, nothing is known.

It may be interesting and necessary to compare these bivalent chromosomes artificially induced in the somatic cell with those observable in the normal maturation division of the germ cell. By reference to the literature hitherto published on the maturation phenomena of Drosophila virilis, a fact became clear viz. that the chromosomes of the primary spermatocyte indicated in Fig. 24 of the paper of Metz ('27) are almost near, in their lateral configuration, to the induced bivalent chromosomes illustrated in Fig. 8 of the present paper. Since the chromosomes of germ cells of $D$. virilis have not been fully worked out due to technical difficulty, and the morphology of bivalent chromosomes has remained mostly unknown, further ccmparison is impossible to make at present.

\section{Discussion}

As shown in the former studies, the ganglia of old larvae of Drosophila about to pupate, reared under the favourable conditions, contain many cells in the process of mitotic division. In the present experiment planned as to retain these ganglion cells for longer time in resting state by means of artificial suppression of the division process due to placing the larvae under unfavourable culture conditions, 
the following evidence was attained: while most cells of the ganglia are persisting in the stage of prophase, some cells show bivalent chromosomes which resulted from pairing of homologous chromosomes, being reduced in number into haploid. This finding seems to evince that mitosis becomes convertible through retardation of cellular activity into meiosis. The present finding offers a suggestion for the analysis of the mechanism of meiosis.

As to the cause and mechanism of chromosome pairing at meiosis, different opinions have been put forward. Darlington ('32, '37) advocated that chromosome threads normally have a strong tendency to associate in pairs in prophase. While in mitotic prophase the tendency is satisfied because the threads are split into double in the resting stage, in meiotic prophase this tendency is unsatisfied due to the precocious initiation of the prophase before the chromosomes split, so that the chromosomes pair with their homologues to satisfy the tendency. This precocity theory of Darlington, therefore, emphasizes the fact that chromosome pairing and synapsis at meiosis depend upon the univalency of the synapting chromosomes. The unified theory proposed little later by Huskins ('33) in a modification of Darlington's precocity theory and based on the assumption that in the last premeiotic division the chromosome splitting is retarded and postponed to take place first at pachytene, so that the tendency of chromosomes to associate in pairs is unsatisfied at leptotene, hence they pair with their homologues. Kuwada ('40) has made another modification of the theory of Darlington, based on his principle of attraction between single (univalent) chromosomes and repulsion between pairs of pairs, by adopting instead of the actual singleness of chromosomes, an apparent singleness due to the lateral fusion of the divided chromo. somes. He said that the physiological conditions of meiosis should differ from those of mitosis and these conditions may cause the chromosome pairing which gives rise to the prophasic prolongation in meiosis.

Sax and Sax ('35) has proposed the hypothesis called the retardation theory which is based on the evidence that the meiotic cycle is a much more leisurely process than the mitotic cycle. They suggest that meiosis results from a retardation of cellular activity rather than precocity in development, as shown by the fact that a much longer time is required for meiosis than for mitosis. The prolongation of prophase at meiosis allows chromosomes to uncoil completely and to pair intimately with gene by gene association. Stebbins ('35), Beasley ('38), Matsuura \& Haga ('40), and some others tend to support the retardation theory from different standpoints. Stebbins ('35), dealing with the structure of the chromosomes, stated that the physiological condition of the nucleus which, in the early prophase of every mitosis, promotes the coming together of chromonemata, is prolonged in the meiotic prophase, so that homologous 
as well as sister chromonemata come together. From the observations regarding the nuclear size, Beasley ('38) advocated that the increase in volume of meiotic prophase nuclei, the decrease in viscosity of the karyolymph, and the comparatively long duration of meiosis, operate harmoniously to allow intimate gene by gene association of homologous chromosomes in the first meiotic prophase. Matsuura \& Haga ('40) supported the view of Sax and Sax ('35) on the basis of experimental study, demonstrating that meiosis is convertible by acceleration of cell activity into mitosis not only in the behaviour of chromosomes but also in their structure.

The finding of the present investigation seems to be one instance which finds its most favourable interpretation by adopting the retardation theory. because of the fact showing that the time required for meiosis is much longer than that required for mitosis. By placing the larvae under unfavourable culture conditions, the mitotic division of ganglion cells is suppressed as is evident by the fact that most cells contained in the ganglia of treated larvae have remained in the resting condition. As the result, the cells persisted in situ in the prophasic condition for longer time than in normal mitosis. The long duration of prophase would permit in the nucleus the homologous chromosomes to become intimately associated in pairs. The particular structure existing in the somatic prophase nucleus of this species characterized by the evidence that every two homologous chromosome threads lie side by side in close proximity, may probably operate effectively for pairing of chromosomes.

Although the results of experimental researches as to the analysis of the mitosis-and-meiosis relationship, one obtained by Matsuura \& Haga and the other by the present study, are thus in favor of the retardation theory of Sax and Sax ('35) in the interpretation of the phenomena, the present author does not expect from these results any final solution for the mechanism of meiosis. It might be probable that the mechanism of meiosis is too complicated to be fully accounted for on the basis of the retardation theory only. However, it may be allowed to say, on the basis of the present investigation, that an attraction between homologous prophase chromosomes is probably present at all times, and that the particular conditions existing in meiotic prophase permit the chromosomes to satisfy the attraction. Perhaps it is a matter of time and some physiological conditions in meiosis that allow chromosomes to associate in pairs.

\section{Summary}

The ganglia of old larvae of Drosophila virilis reared under favourable conditions contain many somatic cells in the process of mitotic 
division. Through the treatment of the larvae under unfavourable culture conditions, the process of the mitotic division in ganglion cells is suppressed, and as a consequence most of these cells remain in the resting stage for longer time than in the normal mitosis. This condition leads to the production of bivalent chromosomes in some ganglion cells, which were produced due to the synaptic pairing of homologous chromosomes, being six in number $(n)$, instead of 12 , the $2 n$ number. The prolongation of the resting stage would permit in the nucleus the homologous chromosomes to become intimately associated in pairs. The evidence presented implies that mitosis becomes convertible by a retardation of the division process into meiosis.

\section{Literature Cited}

Beasley, J. O. 1938. Nuclear size in relation to meiosis. Bot. Gaz. 99: 865-871.

Darlington, C. D. 1932. Recent advances in cytology. (1st Ed.) London.

- 1937. Recent advances in cytology. (2nd Ed.). London.

Huskins, C. L. 1983. Mitosis and meiosis. Nature 132: 62-63.

Kuwada, Y. 1940. Studies on mitosis and meiosis in comparison. I. A morphological analysis of meiosis. Cytologia 11 : 217-244.

Makino, S. 1933. A morphological study of the nucleus in various kinds of somatic cells of Drosophila virilis. (Cytological studies on D. virilis, Pt. I). Cytologia 9: 272-282.

- 1940. On the chromocentre observed through the mitotic cycle of somatic cells in Drosophila virilis. (Cytological studies on D. virilis, Pt. II). Cytologia 10: $283-293$.

Matsuura, H. \& T. Haga 1940. Chromosome studies on Trillium kamtschaticum Pall VIII. On the mitosis-meiosis relationship. Cytologia $10: 382-389$.

Metz, C. W. 1927. Observations on spermatogenesis in Drosophila. Zeits. Zellf. mikr. Anat. $4: 1-28$.

Sax, H. J. \& K. Sax 1935. Chromosome structure and behavior in mitosis and meiosis. Jour. Arn. Arb. 16 : 423-439.

Stebbins, G. L. 1935. Chromosome structure and the mechanism of meiosis in plants. Amer. Nat. $69: 81$. 